\title{
UN ECCE HOMO, DESCONOCIDO, DE LUIS DE MORALES EN HUNGRÍA
}

Aunque Morales es uno de los pintores españoles más conocidos y queridos de cuyas obras ya se había hecho una gran exposición a principios de siglo en el Prado ${ }^{1}$, y después de la monografía de Bäcksbacka (1962) se publicaron más artículos sobre él y catálogos de sus obras que han precisado los conocimientos sobre el maestro. Sin embargo, hasta hoy se ignora la fecha de su nacimiento y falta una nueva monografía con un catálogo de sus obras ${ }^{2}$. Según el libro de Palomino se sabe que su maestro fue, en Sevilla, Pedro de Campaña ${ }^{3}$. Su influencia y la de los pintores flamencos con su técnica minuciosa es considerable sin duda en la técnica de pintar de Morales.

Al mismo tiempo, no se puede excluir un viaje suyo en Italia, aunque es posible, como supuso ya Trapier, que le bastaría estudiar las pinturas leonardescas de El Escorial que tomó por modelo para su manera del esfumato ${ }^{4}$. En su grandiosa obra, aunque algunos retablos documentados puedan reconstruirse, los muchos ejemplos de los Ecce Homo que sirvieron para las tablas centrales de los retablos más pequeños, hacen suponer que solamente se conservó una parte de la obra de Luis de Morales.

Está documentado que tuvo un taller significado donde trabajaba con él su hijo, llamado Cristóbal. Cuando se destaca una nueva obra suya es necesario sopesar la autoría. Por nuestra parte, identificando un Ecce Homo, aunque de muy alta cualidad, me enfrenté con el mismo problema. En Hungría, en la coleción eclesiástica se encuentra desde 1844 este Ecce Homo de medio cuerpo con la posición de la cabeza casi de frente mirando hacia el espectador, que según mis conocimientos, por la dirección de la mirada de Cristo y la posición de la cabeza, es único en la obra de Morales (Fig. 2).

La pintura fue ya conocida desde 1950 por los investidores húngaros que la habían considerado como una copia de la pintura que hay en el depósito del Museo de Bellas Artes de Budapest y que menciona en su catálogo Pigler como obra de un seguidor de Morales (Fig. 3) y es verdad que tiene una composición bastante parecida ${ }^{5}$ pero sus colores son diferentes. En-

${ }^{1}$ Exposición de obras del Divino Morales celebrada en el Museo del Prado de Madrid desde el 1 al 31 de mayo de 1917. Madrid, 1917.

${ }^{2}$ La literatura no exhaustiva después de la monografía de I. Bäcksbacka: Luis Morales, Helsinki, 1962; Gaya Nuño, J. A. Luis de Morales, Madrid, 1961 (Bäcksbacka no se menciona verosímilmente por la concordancia temporal); A. R. G de Ceballos.: Nuevas pinturas de Luis Morales. Archivo Español de Arte, 1973, 181-4, 69-71; Pérez Sánchez, A. E.: El retablo de Morales de Arroyo de la Luz. Dirección General de Bellas Artes. Madrid, 1974; Torres Pérez, J. M.: Las complejas fuentes de inspiración en la pintura de Luis Morales. Revista de Estudios Extremeños, Badajoz, 1975, XXXI, 163-180; García Mogollón, F.: En torno al retablo de la iglesia parroquial de Arroyo de la Luz (Cáceres), Estudios dedicados a Carlos Callejo Serrano. Cáceres, 1979; Diéguez Luengo, E.: La Virgen con los dos Santos Juanes. Morales y el número de oro. Estudios dedicados a Carlos Serrano. Cáceres, 1979; Solís Rodríguez, C.: El retablo de Morales en Puebla de la Calzada. Bellas Artes, 6, 1975, 43 (12-14); Solís Rodríguez, C.: Luis de Morales. Revista de Estudios Extremeños, 34, 1978, 49-137; González Rodríguez, A.: El Morales de Villanueva de la Serna. Badajoz, 1984. Díaz Padrón y M. Padrón Mérida, A.: Miscelánea de pintura española del siglo XVI. Archivo Esañol de Arte, n. ${ }^{\circ} 223$, 1983, 193-219; Tablas de Luis de Morales en Valencia de Alcántara y San Martín de Trevejo, Mérida, 1988. González Rodríguez, A.: Aproximación biográfica en Tablas de Luis de Morales en Alcántara y San Martín de Trevejo, Mérida, 1988: A. E. Pérez Sánchez: Un nuevo y curioso documento sobre Morales. Archivo Espñol de Arte, 1977, 199, 313-316; F. Benito Domenech: Museo del Patriarca de Valencia, 1991. Nuevas tablas del Divino Morales y del Maestro de Portillo. Informes y trabajos del Instituto Central de la Restauración y Conservación de Obras de Arte. Ministerio de Educación y Ciencia. Dirección General de Bellas Artes. Madrid, núm. 7, 1968, 17-20. Barcelona, Ana Avila in L'Epoca dels Genis. Renaixement-Bamoc. 1990, n. ${ }^{\circ} 11,122-4$.

3 Palomino de Castro y Velasco, A.: El parnaso español pintoresco laureado. Londres. 1774, 20, pp. 13-14.

${ }^{4}$ E. du Gué Trapier: Luis de Morales y la influencia leonardesca en España. Revista de Estudios Extremeños, IX, 1953, 653, 684 (ante todos 668-69 pp.).

${ }^{5}$ A. Pigler: Katalog der Galerie Alter Meister, I. Budapest, 1967, 464, inv. n. ${ }^{\circ} 9.782$, o. sobre lienzo, 65,5x50,3 cm «copia después en diferentes variantes del conocido tipo de Luis de Morales». 


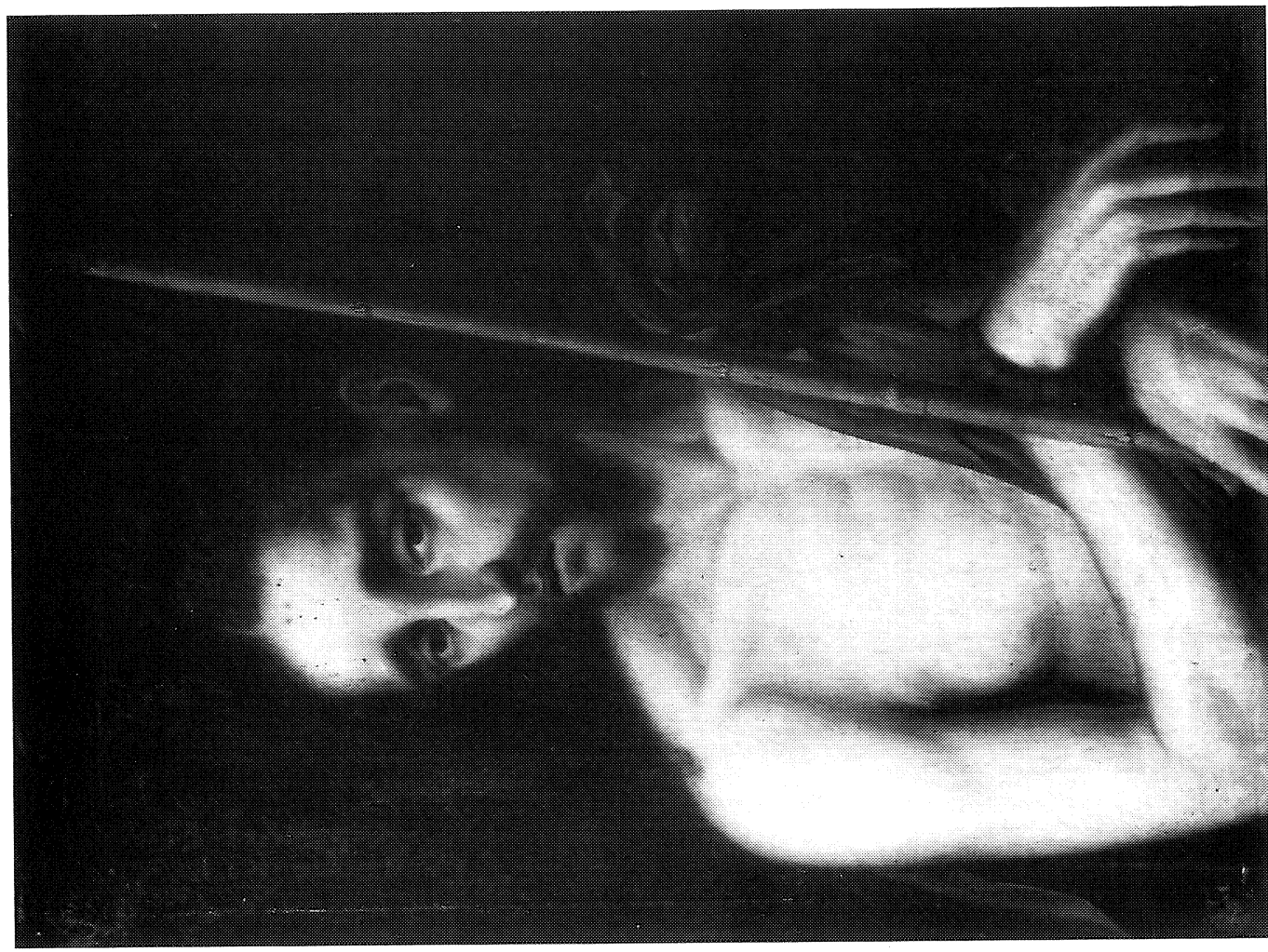


tonces se consideró que la obra aquí estudiada estaba muy repintada, por lo que no podía precisarse su fecha ${ }^{6}$.

La pintura fue trasladada al Museo de Bellas Artes de Budapest hace dos años, $\mathrm{y}$ tras su estudio pensamos que merecía ser publicada ${ }^{7}$.

La tabla es de nogal. Sus medidas son un poco irregulares: $634-635 \times 472-474 \mathrm{~mm} \times 8 \mathrm{~mm}$. Todos los cantos de la tabla son originales, es decir la capa de la imprimación cubre el canto actual. La pintura fue limpiada hace algunos años. Sobre la frente de Cristo, en el fondo a la izquierda, aparece una ranura vertical que, en la radiografía, se ve sobre toda la tabla, y al lado de ésta retoques no ajustados, por ejemplo, en el brazo de Cristo.

La radiografía no muestra cambios en la composición. Fue más interesante y de más información la reflectografía de infrarrojos donde es visible un dibujo de gran técnica. Las partes más atractivas son estos dibujos preparatorios, de lápiz de plata, en el hombro de Cristo bajo el manto, en las manos, donde la posición de los dedos, pulgar y meñique son diferentes de los pintados.

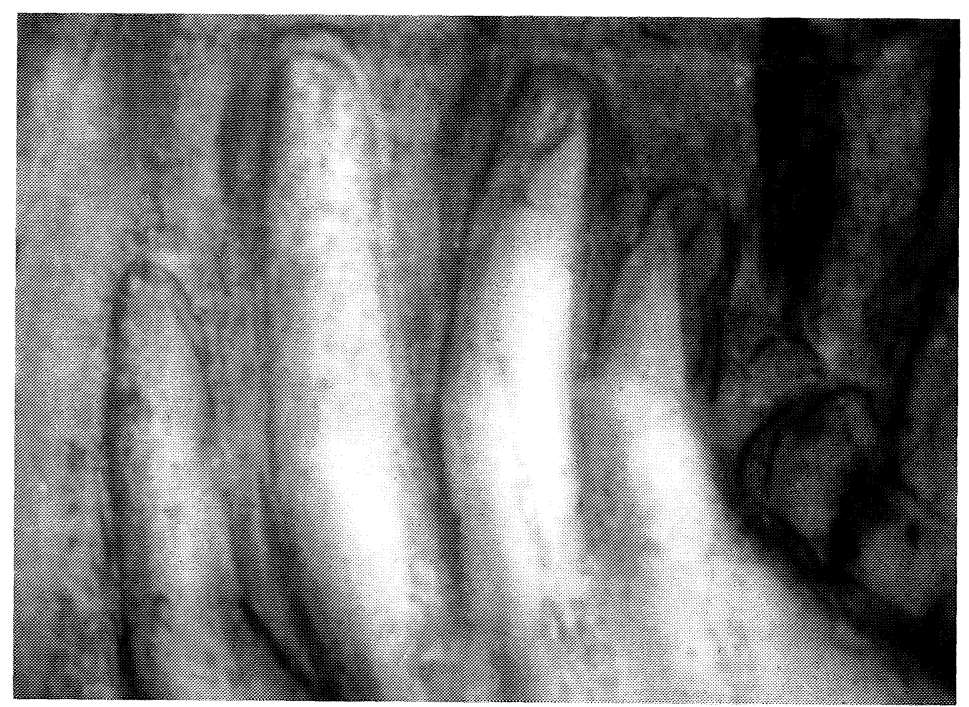

Fig. 4. Luis de Morales. Ecce Homo, detalle de la mano. Hungria. Colección arzobispal.

El tamaño actual de esta tabla es original porque muestra también una línea horizontal del dibujo subyacente, en la mano izquierda de Cristo, que se ve bajo la reflectografía de infrarrojos que muestra que terminó aquí la composición según el maestro, y la mano izquierda estaba así ya en su concepción cortada.

En contraste con el fondo negro aparece Cristo con ojos pardos, pelo castaño y manto del típico color azul-verdoso de Morales. En su frente se ven gotas de sangre y lágrimas en su cara. Delante del hombro izquierdo sostiene el cetro de caña y sus muñecas están trabadas con una cuerda de estopa.

En la comparación crítica del estilo de la obra he buscado ante todo las analogías entre los Ecce Homos de Morales. El tipo de Cristo aguileño, mentón y nariz agudos es parecido al Cristo de figura

${ }^{6}$ En el Departamento de la Documentación del Museo de Bellas Artes se lee la opinión de I. Fenyö, que la pintura aquí publicada sea una copia distinta de la pintura del Museo de Bellas Artes de Budapest, aunque el manto de Cristo en Budapest es rojo. La pintura está fuertemente repintada y por eso es difícil de apreciar.

${ }^{7}$ La pintura fue estudiada en el Museo de Bellas Artes por el restaurador Miklós Szentkirályi y las técnicas de Sándor Szilágyi. 
entera de la tabla central de la iglesia parroquial de Higuera la Real ${ }^{8}$. Allí Cristo, como casi siempre en los Ecce Homos mira hacia abajo no hacia el espectador. Morales carcteriza igualmente el dolor en Higuera la Real y en la Ecce Homo conservado en Hungría: enmarcan la cejas en el centro de la frente y presentan los labios entreabiertos. El modelado de los ojos y pómulos, con sfumato, muestran la influencia de los seguidores lombardos de Leonardo y no el tipo inspirado en el Piombo Cristo portando la cruz que en Morales es también más toscano y en sus maneras más seco ${ }^{9}$.

El Ecce Homo del Museo de Lisboa también de figura entera, que según Bäcksbacka es idéntico a la obra de la Casa del Capítulo de Évora, es también parecido a la nuestra, aunque allí los labios están cerrados y la mirada también se dirige hacia abajo, pero el carácter de la cara es parecido a nuestro Cristo. La manera de pintar de la obra de Lisboa es más fluida y por su técnica proviene de los años de 1570, según Bäcksbacka ${ }^{10}$.

El tipo de cabeza de Cristo de Hungría, aunque está más transfigurada (espiritualidad), es muy parecida a la cabeza inspirada en Michelangelo de San Juan del Prado ${ }^{11}$. Las luces sobre los labios caracterizados con pequeñas líneas paralelas, el pelo, la barba están pintados con hebras de cabello imitado el natural. La técnica, muy flamenca, también se puede advertir en las dos pinturas semejantes en sus tamaños; y ambas provienen de los años 1565-70 de Morales.

Ultimamente, Serrera publicó la nueva adquisición del Prado, la Adoración de los Reyes Magos donde el Rey Melchor, en la mitad, de su tipo aparece también como el Cristo del Ecce Homo aquí publicado ${ }^{12}$. La manera de pintar las caras, el tono de las carnaciones, el modelado alrededor de los ojos son similares a las cejas de los santos de Morales en la colección Masaveu, San Francisco de Asís y San Pedro de Verona ${ }^{13}$. Con relación a estas tablas, Serrera supuso que eran alas de un retablo pequeño para devoción privada y en el centro también tendría un Ecce Homo. La procedencia común del convento de las Comendadoras de Santiago, de Toledo, de estas tablas, con un Ecce Homo, publicado también en el catálogo de la colección Masaveu, y las dimensiones de las alas que son más altas y estrechas sugieren que Serrera tiene razón en esta reconstrucción ${ }^{14}$.

En su origen la obra de Hungría, verosimilmente, también sería talla central de un tríptico por su tamaño igual al Ecce Homo del tríptico de la Catedral de Sevilla, de Morales ${ }^{15}$.

Allí sobre las alas se ve la Madre Dolorosa y San Juan. Pero en las obras de Morales sería más probable una Dolorosa o, según el concepto contrarreformista, un santo en penitenia con un Ecce Homo ${ }^{16}$. Al igual que en la tabla de Hungría no se aprecia el ajuste de las puertas,aunque es verdad que éste, originalmente, se encontraba sobre el marco original, que no existe en nuestro tiempo ${ }^{17}$.

La composición del Ecce Homo de Hungría, la posición de las manos con el cetro de caña y el detalle del manto atado en el hombro es análogo al Ecce Homo, conocido, de la colección madrileña de Ezequiel de Selgas del que se diferencia solamente en la dirección de la cabeza de Cristo ${ }^{18}$. En esta obra, según Escobar es «de la flor de Morales» ${ }^{19}$, tres dedos de la mano que

${ }^{8}$ I. Bäcksbacka, op. cit., 1962, 83-5, cat. 19:2, 158-9, fig. 42.

9 Vid., por ejemplo, el Ecce Homo del Museo de Dresden: I. Bäcksbacka, op. cit., 1962, 66, cat. 16, 154, fig. 22.

${ }^{10}$ I. Bäcksbacka, op. cit., 1962, 100, cat. 35, 166, fig. 74 .

11 I. Bäcksbacka, op. cit., 1962, 95, cat. 22, 162, fig. 58, menciona como obra auténtica. J. J. Luna: Guía actualizada del Prado. Una historia de la pintura a través de las obras del Museo. Madrid, 1984, 34, la cree de taller de Morales.

12 J. M. Serrera en el cat.: Un mecenas Póstumo. El Legado Villaescusa. Adquisiciones 1992-93. Museo del Prado. Madrid, 1993, 36-39.

${ }_{13}$ J. M. Serrera en el cat. Obras maestras de la Colección Masaveu. Museo del Prado. Madrid, 1989, n. ${ }^{\circ}$ 12, n..$^{\circ}$ 13, $52-55$.

14 J. M. Serrera an el cat. Obras maestras de la Colección Masaveu. Museo del Prado. Madrid, 1989. 54. Vid. el mismo Ecce Homo en I. Bäcksbacka, op. cit., 94, 1962, cat. 23, 162, fig. 59.

${ }^{15}$ I. Backsbacka, op. cit., 1962, 64, cat. 13, 153, fig. 19.

16 F. Zeri: Pittura e Contrariforma. L'Arte senza tempo di Scipione da Gaeta. Torino, 1957, 65.

${ }^{17}$ El marco actual de la pintura proviene de los años 1840, de tiempos de la llegada de la obra a Hungría.

18 I. Bäcksbacka, op. cit., 1962, 122, cat. 70, 177, fig. 116.

${ }^{19}$ D. Berjano Escobar: El Divino Morales, Madrid, 1926, 109, Add. IV. 
sostiene la caña están también cortados. Concuerda la obra aquí estudiada con el Ecce Homo del tríptico llamado de Oñate, descontando también la posición de la cabeza de Cristo ${ }^{20}$. Comparando la manera de pintar las telas, sobresale la semejanza con el San Jerónimo penitente, de Dublín, donde el nudo del manto es también muy plástico ${ }^{21}$.

Las analogías tipológicas y estilísticas nos dan la posibilidad de datar la pintura, aquí estudiada dentro de la obra de Morales, con bastante seguridad, alrededor de 1570. Los puntos de referencia de la datación son el grandioso retablo de 1566 bien documentado en Higuera la Real ${ }^{22}$ y las tablas muy parecidas de San Francisco de Asís y San Pedro de Verona publicadas recientemente por Serrera ${ }^{23}$ fechada por él hacia 1565 y la Adoración de los Reyes Magos, nueva adquisición del Prado, publicada por el mismo autor, datada entre 1570-75, época de esplendor de Morales ${ }^{24}$.

La paternidad de Morales del Ecce Homo de Hungría la atestiguan no sólo detalles como el pelo, la barba, las cejas, los ojos, las pestañas, las gotas de sangre y las lágrimas pintadas con una técnica excelente ${ }^{25}$, sino también la manera de pintar, plástica y típicamente metálica el manto y su color azul verdoso típico de Morales, que forma un contraste característico con el fondo negro. Otro motivo es la posición de la cabeza de Cristo, su mirada, que es muy personal, dirigida hacia el espectador que está frente a él y que es totalmente única en la obra de Morales ${ }^{26}$. Los tipos mirando fuera del cuadro hacia el observador son por lo general los San Juan Bautistas, por ejemplo, sobre el altar en Valencia de Alcántara o Pilato en los Ecce Homos con más personajes, como se ve en la obra de la Hispanic Society ${ }^{27}$ de Nueva York, o el Cristo resucitado, en la colección de J. Salvans Piera, en Barcelona ${ }^{28}$. Hay personajes atípicos como la Zelemi de los Apócrifos en la Sagrada Familia de Nueva York ${ }^{29}$, o el rey Baltasar en la nueva adquisición del Prado. Este último personaje, casi hace contacto dirigiéndose al espectador y atrae con su mirada, al devoto. Los Ejercicios Espirituales se divulgaron a través de los jesuitas en torno a Badajoz ${ }^{30}$. Los jesuitas fueron llamados allí por el obispo pacense Cristóbal Rojas y Sandoval y por ellos fueron conocidos los Ejercicios Espirituales de San Ignacio de Loyola. En tiempo del obispo Juan de Ribera, mecenas de Morales se extendió, gracias a los escritos de Juan de Ávila y Fray Luis de Granada, un espiritualismo no siempre ortodoxo. Esta mirada del Ecce Homo aquí publicado, sin par en la obra de Morales, también puede relacionarse con esta corriente espiritual más personal e individual del jesuitismo y según esta referencia la datación se concreta hacia 1570.

${ }^{20}$ I. Bäcksbacka, op. cit., 1962, 110. cat. 45, 168, fig. 89.

${ }^{21}$ R. Mulcahy: Spanish Paintings in the National Gallery of Ireland. Dublín, 1988, 40, Inv. n. ${ }^{\circ}$ 1. fig. 26.

${ }^{22}$ I. Bäcksbacka, op. cit., 1962, 83-86.

23 J. M. Serrera en el cat. Obras maestras de la Colección Masaveu. Museo del Prado. Madrid, 1989, 54.

${ }^{24}$ J. M. Serrera en el cat. Un mecenas Póstumo. El legado de Villaescusa. Adquisiciones 1992-93. Museo del Prado. Madrid, 1993, 39.

${ }^{25}$ Sobre la técnica de Morales vea E. du Gué Trapier: «Luis de Morales y las influencias leonardescas en España», en Revista de Estudios Extremeños, T. IX, 1953, 653-684, en p. 677: «El realismo con que ha pintado la lágrima en la mejilla de Cristo, las largas pestañas de pinceladas separadas los detalles de las barbas indica un pintor de escuela flamenca como su modelo aunque ciertos lombardos trataron el tema del mismo modo».

Según su calidad Backsbacka, op. cit., 1962, 118, cat. A 17, p. 188, fig. 153 cita un Ecce Homo como obra del taller en el Catálogo Las Edades del Hombre (El Contrapunto y su Morada), Salamanca, 1993, cat. 29, p. 79 en el Museo Catedralicio de Ávila fue atribuido a Morales. En la subasta de Sotheby's Peel, Madrid, Pintura antigua y escultura, el 27 de octubre, 1992, cat. 12. Cristo atado a la columna fue expuesta como obra de Morales. Las dos últimas obras no tienen tan alta calidad como la aquí publicada.

${ }^{26}$ Sobre un Ecce Homo no muy conocido, datado también alrededor de 1570, Vid. en el cat. L'Epoca dels Genis. Renaixement-Barroc. A. Ávila en cat. $\mathrm{n} .{ }^{\circ} 11,122-124$. Barcelona, 1990

${ }^{27}$ I. Bäcksbacka, op. cit., 1962, cat. 24, p. 162-3, fig. 60; Trapier, E. du Gué Luis de Morales and leonardesque ifluence in Spain, New York, 1953, p.

${ }^{28}$ I. Bäcksbacka, op. cit., 1962, cat. 64, 175, fig. 108.

${ }^{29}$ I. Bäcksbacka, op. cit., 1962, cat. 72, 178, fig. 118.

${ }^{30}$ Serrera in cat. Un Mecenas Póstumo. El Legado Villaescusa. Adquisiciones, 1992-93, 36-39. 
Las circunstancias de la llegada de esta obra a Hungría son desconocidas. Aparte del dato que desde el 18 de octubre de 1844 está registrado en el fundus instructus, según la inscripción que se lee sobre el reverso de la pintura, en la colección arzobispal donde hoy se conserva. Suponemos que la obra fue sacada, durante los años de la invasión francesa, de su lugar de origen y transportada fuera de España ${ }^{31}$.

En la colección de Luis Felipe existió un Ecce Homo, de Morales que en sus medidas es idéntico a esta obra, pero aquél fue ya identificado justamente con la obra de la Hispanic Society of America ${ }^{32}$. No se pudo identificar tampoco con seguridad con otras obras mencionadas por Bäcksbacka en diferentes subastas y colecciones, dado que las obras se mencionaban a menudo, en el siglo pasado, sin medidas. Esta obra, inédita sería de algún retablo sacado de las iglesias cercanas a Badajoz desde los años 1800 y que por sus pequeñas dimensiones fueron transportados sin problemas, y se vendieron las tablas separadamente ${ }^{33}$. No se puede excluir tampoco que llegara a Hungría como un regalo de la Iglesia de España.

EVA NYERGES

Museo de Bellas Artes de Budapest

\section{ANOTACIONES SOBRE LA VIDA Y LA OBRA DEL ARQUITECTO SIMÓN GARCÍA}

Simón García ha pasado a la historia del arte como «tratadista» por su «Compendio de Arquitectura y Simetría de los Templos» redactado entre 1681 y 1683, que nunca llegaría a ver la imprenta en vida del autor ${ }^{1}$. El profesor Bonet reivindicó en su día la importancia de este manuscrito no sólo por habernos transmitido un texto anterior de Rodrigo Gil de Hontañón, sino como síntesis de los conocimientos teóricos y prácticos sobre el arte de construir que podía llegar a alcanzar un «buen maestro de arquitectura» en el siglo XVII. En su opinión, Simón García conjuga una información teórica de raíz clasicista con la tradición geométrica medieval, y al mismo tiempo se muestra al tanto de las nuevas corrientes de su tiempo ${ }^{2}$.

Por contraste apenas se sabe otra cosa de este personaje que lo que él mismo nos dice en su tratado. Será necesario un estudio más profundo de la actividad artística desarrollada en Sala-

\footnotetext{
31 No existe en nuestros días el fundus instructus.

32 J. Baticle-C. Marinas: La Galerie espagnole de Louis-Philippe au Louvre, 1838-1848, cat. 144, 107. Según la descripción de A. de Circourt (1838) L'Ecce Homo de Morales en la col. de Louis Philippe representa también Pilato que posibilidad para la identificación de esta con la col. de Nueva York Hispanic of America.

${ }^{33}$ Veáse, or ejemplo, la historia del retablo de La Virgen del pajarito, ${ }^{27}$ I. Bäcksbacka, op. cit., 1962, 24. La obra fue trasladada entre 1834-39 de la iglesia de la Concepción de Badajoz. Gaya Nuño, J. A.: Luis de Morales, Madrid, 1961, n. ${ }^{\circ}$ 2 , p. 41 y fue vendida en 1834 .

${ }^{1}$ El Colegio Oficial de Arquitectos de Valladolid publicó en 1991 una nueva edición facsímil, con los estudios de los profesores Bonet Correa y Chanfón Olmos que acompañaban la primera edición completa conocida, aparecida en Churubusco (México) en 1979. Camón Aznar publicó parte del manuscrito en una edición de la Universidad de Salamanca del año 1941. Anteriormente Eduardo de Mariátegui había dado a conocer los capítulos que, en su opinión, correspondían al original de Rodrigo Gil de Hontañón («Compendio de Arquitectura», en Arte en España, t. VII, Manuel Galiano, Madrid, 1868).

${ }^{2}$ Bonet Correa, A., «Simón García tratadista de arquitectura», en el estudio introductorio a la edición facsímil de 1991, pp. 13-18.
} 